\title{
Hydroxyapatite-doped polycaprolactone nanofiber membrane improves tendon-bone interface healing for anterior cruciate ligament reconstruction
}

This article was published in the following Dove Press journal:

International Journal of Nanomedicine

7 December 2015

Number of times this article has been viewed

Fei $\operatorname{Han}^{1, *}$

Peng Zhang ${ }^{2, *}$

Yaying Sun ${ }^{2}$

Chao Lin'

Peng Zhao'

Jiwu Chen ${ }^{2}$

'Shanghai East Hospital, The Institute for Biomedical Engineering and Nanoscience, Tongji University School of Medicine, Tongji University, ${ }^{2}$ Department of Sports Medicine, Huashan Hospital, Fudan University, Shanghai, People's Republic of China

*These authors contributed equally to this work
Correspondence: Peng Zhao Room 220, Shixun Building, 1239 Siping Road, Shanghai East Hospital, The Institute for Biomedical Engineering and Nanoscience, Tongji University School of Medicine, Tongji University, Shanghai, 200092, People's Republic of China

Tel +862 2I 65988029

Fax +86 2I 6598 3706; Extn 0

Email zp@tongji.edu.cn

Jiwu Chen

Department of Sports Medicine, Huashan Hospital, Fudan University, 12 Wulumuqi Middle Road, Shanghai, 200040, People's Republic of China Email jiwuchen@gmail.com

\begin{abstract}
Hamstring tendon autograft is a routine graft for anterior cruciate ligament (ACL) reconstruction. However, ways of improving the healing between the tendon and bone is often overlooked in clinical practice. This issue can be addressed by using a biomimetic scaffold. Herein, a biomimetic nanofiber membrane of polycaprolactone/nanohydroxyapatite/collagen ( $\mathrm{PCL} / \mathrm{nHAp} / \mathrm{Col}$ ) is fabricated that mimics the composition of native bone tissue for promoting tendon-bone healing. This membrane has good cytocompatibility, allowing for osteoblast cell adhesion and growth and bone formation. As a result, MC3T3 cells reveal a higher mineralization level in PCL/nHAp/Col membrane compared with PCL membrane alone. Further in vivo studies in ACL reconstruction in a rabbit model shows that PCL/nHAp/Col-wrapped tendon may afford superior tissue integration to nonwrapped tendon in the interface between the tendon and host bone as well as improved mechanical strength. This study shows that PCL/nHAp/Col nanofiber membrane wrapping of autologous tendon is effective for improving tendon healing with host bone in ACL reconstruction.
\end{abstract}

Keywords: nanohydroxyapatite, polycaprolactone, nanofiber, tissue engineering, anterior cruciate ligament reconstruction

\section{Introduction}

Anterior cruciate ligament (ACL) rupture is one of the most serious knee injuries in those athletes participating in high-risk sports. ${ }^{1-3}$ From a clinical point of view, hamstring tendon autograft is a well-accepted surgical choice for ACL reconstruction because of advantages such as low possibility of donor-site morbidity and degenerative joint disease as well as high extension strength. ${ }^{4-6}$ An ACL reconstruction surgery normally involves penetrating a tendon autograft through bone tunnel and anchoring it between the tibia and the femur. A critical clinical issue is that the healing time of the tendon in the bone tunnel is relatively long as a result of the lack of a suitable biointerface between the tendon and bone for efficient integration. For that reason, clinical healing efficacy of tendon is often poor. Thus, there is a need to develop an effective method for tendon-bone healing in ACL reconstruction.

Tissue engineering offers a novel strategy for promoting tissue reconstruction such as osteoregeneration. ${ }^{7}$ By this method, nanostructured scaffolds are designed and fabricated to provide an artificial biointerface by mimicking the extracellular matrix. In the past 2 decades, these nanoscaffolds containing biodegradable materials such as polycaprolactone (PCL), chitosan, and collagen (Col) present a great potential for ACL repair. ${ }^{8-10}$ A co-electrospun based on PCL and Col onto opposite ends of a mandrel was fabricated to create a scaffold with three regions to repair muscle-tendon junctions. ${ }^{11}$ 
In another work, a new silk scaffold was prepared by incorporating microporous silk sponges into silk mesh to mimetic microscale structures of ACL extracellular matrix. ${ }^{12}$ Recently, a low-crystallinity hydroxyapatite-doped fibroin scaffold was developed for bone-tissue interface repair and osteogenesis. ${ }^{13}$ Our work showed that PCL/nanohydroxyapatite (nHAp) composites are efficient for in vivo osteoconduction of stem cells. ${ }^{14}$ These current studies suggest that the design of functional biomimetic scaffolds for osteoconduction is useful for improving tendon-bone healing in ACL reconstruction.

The aim of this work was to fabricate osteoconductive nanofiber membranes based on PCL and nHAp to promote tendon-bone interface integration (Figure 1). Compared with Col or silk sponge, PCL-based membrane has high potential for clinical translation due to easy fabrication, large-scale preparation, and good biocompatibility. This biomimic membrane consists of nHAp and Col-doped PCL nanofiber. We hypothesized that, by encapsulating the membrane around autologous hamstring tendon, the tendon and bone could be efficiently integrated together. Herein, we described the fabrication of PCL-based nanofiber membrane and evaluated its cytocompatibility using MC3T3 cells. In vivo evaluation on the membrane for promoting tendon-bone healing was also conducted by wrapping nanofiber membrane on autologous tendon and implanting the tendon in a rabbit model.

\section{Materials and methods Materials}

PCL $(\mathrm{Mw}=80 \mathrm{kDa}), N, N^{\prime}$-dimethylformamide and calf skin Col type I were obtained from Sigma-Aldrich (St Louis, MO, USA). nHAp were prepared by a reaction of $\mathrm{Ca}\left(\mathrm{NO}_{3}\right)_{2} \cdot 4 \mathrm{H}_{2} \mathrm{O}$, $\left(\mathrm{NH}_{4}\right)_{2} \mathrm{HPO}_{4}$, and $\mathrm{NH}_{4} \mathrm{HCO}_{3}$ using a nanoemulsion method. ${ }^{15}$

\section{Fabrication of nHAp-doped PCL nanofiber membrane}

Nanofiber membranes were prepared by a routine electrospinning method. ${ }^{16}$ In brief, a solution of PCL (0.3 g) and nHAp $(0.2 \mathrm{~g})$ in dimethylformamide/chloroform solvent $(3 / 1, v / v, 4 \mathrm{~mL})$ was gently mixed with Col type I (0.15 g) solution in hexafluoroisopropanol $(2 \mathrm{~mL})$ by stirring overnight at 300 rotations per minute. Ultrasound was used to keep nHAp in a dispersion state. This resulting PCL/nHAp/ Col solution was then delivered through a needle at a constant flowing rate $(0.5 \mathrm{~mL} / \mathrm{h})$, derived by a pump (LSP01-1A, Longer Pump Inc., Baoding, People's Republic of China), for nanofiber generation at a voltage of $15 \mathrm{kV}(\mathrm{ZS}-60 \mathrm{kV} / 2 \mathrm{~mA}$, Rixing Electric Inc. Shanghai, People's Republic of China). The environmental condition of spinning device was set at $35^{\circ} \mathrm{C}$ and $30 \%$ humidity. An aluminum foil plate was used as a collector for collecting the nanofiber and located at $15 \mathrm{~cm}$ far from the needle tip.

Electrospinning nanofiber was coated with gold (JEOL JFC-1600 Auto Fine Coater, Tokyo, Japan) and visualized by scanning electron microscopy (SEM, Vega3, Tescan Co. Ltd., Brno, Czech Republic) at $20 \mathrm{kV}$. The diameter of the fiber was measured with ImageJ software (TS5136MM, Tescan Co. Ltd., Seoul, South Korea). Chemical analysis of PCL/nHAp/Col nanofiber membrane was conducted using attenuated total reflection Fourier-transform infrared meter (Tensor 27, Bruker, Woodlands, TX, USA) over a range of wavelength between 4,000 and $400 \mathrm{~cm}^{-1}$. Pore size was measured by bubble point method. The pores of the specimens were filled with water, and then, the water was displaced from the pores by pressurized nitrogen gas. The diameter of pores could be calculated based on the pressure
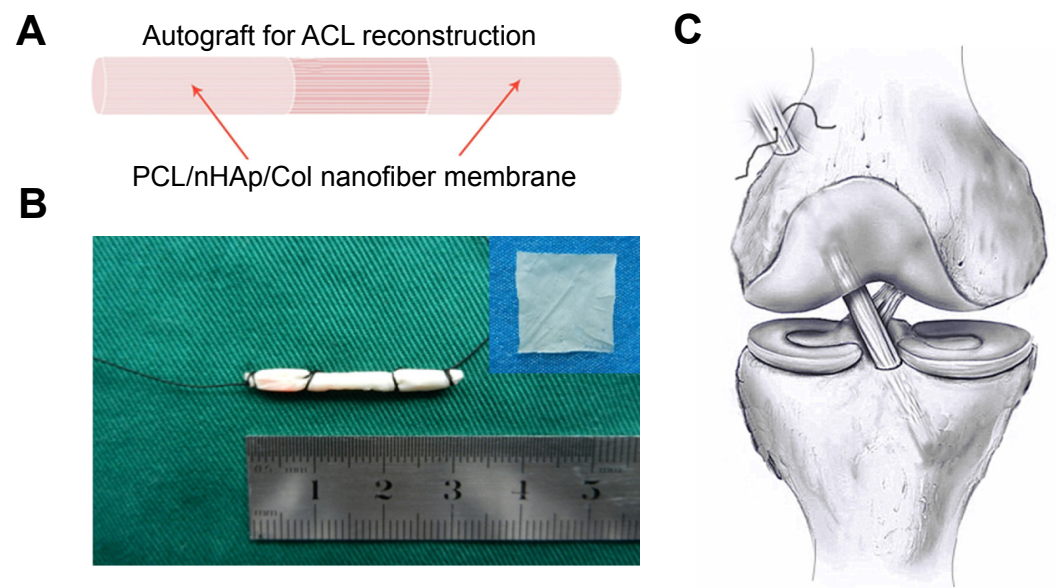

Figure I Design of nanofiber membrane for $\mathrm{ACL}$ reconstruction.

Notes: (A) Illustration of PCL/nHAp/Col nanofiber membrane. (B) PCL/nHAp/Col nanofiber membrane-wrapped tendon. (C) Implantation of the membrane-wrapped tendon into femur and tibia bone.

Abbreviations: $\mathrm{ACL}$, anterior cruciate ligament; Col, collagen; $\mathrm{nHAp}$, nanohydroxyapatite; $\mathrm{PCL}$, polycaprolactone. 
gradient needed to displace the water from the pores in this measurement. ${ }^{17}$ The porosity was estimated by weight and volume of the sample. ${ }^{18}$

\section{Osteoblast cell culture}

MC3T3-E1 murine preosteoblast cell line was purchased from the Type Culture Collection of Chinese Academy of Sciences (Shanghai, People's Republic of China). The collected aluminum foil nanofiber was cut into a wafer at $1.3 \mathrm{~cm}$ in diameter and soaked in $70 \%$ ethanol for 30 minutes. The wafer was first placed into a well of a 24 -well plate for 3 hours drying at room temperature and then placed in each well of a 24-well plate. After twice washing of the wafer with sterile phosphatebuffered saline (PBS), MC3T3-E1 cells $\left(2 \times 10^{4}\right.$ cells/well $)$ were seeded on the wafer in complete medium $(0.5 \mathrm{~mL} /$ well) containing 10\% fetal bovine serum (Gibco, Thermo Fisher Scientific, Waltham, MA, USA) and 1\% penicillin and streptomycin solution (Gibco, Thermo Fisher Scientific, Waltham, MA, USA).

\section{Cell morphology}

According to the method mentioned earlier, MC3T3 cells were cultured for 1, 4, and 7 days. For observation, the cells were washed twice with PBS and immersed in PBS containing $1 \%$ glutaraldehyde for 12 hours. They were then dehydrated in ethanol solution at concentrations of $20 \%, 40 \%$, $60 \%, 80 \%$, and $100 \%$ and finally dried at $25^{\circ} \mathrm{C}$. The sample was gold sputtered under vacuum and visualized under SEM (Vega3, Tescan Co. Ltd., Brno, Czech Republic).

\section{Cell proliferation assay}

MC3T3 cells $\left(2 \times 10^{4} /\right.$ well $)$ were seeded in PCL or PCL/ nHAp/Col membrane in the well of a 24-well plate. Each group had five samples. Cell proliferation was monitored after 1, 3, 5, and 7 days by Cell Counting Kit-8 assay (Dojindo Molecular Technologies, Inc., Kumamoto, Japan). In brief, the cells were treated with $500 \mu \mathrm{L}$ of medium and $50 \mu \mathrm{L}$ of WST- 8 solution, then incubated at $37^{\circ} \mathrm{C}$ for 2 hours; $200 \mu \mathrm{L}$ of the resulting solution from each well was transferred to a 96-well plate. Optical density value at $450 \mathrm{~nm}$ was recorded with a microplate reader (Victor X, PerkinElmer Inc., Waltham, MA, USA).

\section{Mineralization}

Alizarin Red S (ARS) is a dye which selectively binds calcium salts and is a widely used stain for calcium deposit, an indicator of mature osteocytes. ARS staining was used to detect and quantify mineralization. ${ }^{19}$ After 5 and 10 days culture, MC3T3 cells in nanofiber membrane were washed with PBS three times (30 seconds each time) and fixed in $4 \%$ paraformaldehyde for 1 hour. After washing again with double-distilled water, the cells were stained with ARS solution for 30 minutes at $25^{\circ} \mathrm{C}$. The cells were then washed with water, and were desorbed with $10 \%$ cetylpyridinium chloride (Sigma-Aldrich, St Louis, Missouri, USA). for 1 hour. The solution was collected and optical density was recorded at $540 \mathrm{~nm}$ in a spectrophotometer (Multiskan MK3, Thermo Fisher Scientific, Waltham, MA, USA).

\section{Animal study design and surgical procedure}

Twenty-four skeletally mature female New Zealand White rabbits (12 weeks old, $2.4 \pm 0.3 \mathrm{~kg}$ ) were used. These rabbits were divided into two groups (PCL/nHAp/ Col group and PCL group) with six rabbits in each group. The animal study was approved by The University of Fudan Institutional Animal Care and Use Committee and conducted according to the Guide for the Care and Use of Laboratory Animals of Fudan University. A diagrammatic representation of the procedure of ACL reconstruction surgery is shown in Figure S1. Briefly, under general anesthesia, the bilateral limbs were disinfected before skin incisions were made. The lateral parapatellar arthrotomy was used after exposure of right knee joint of the rabbit. All the soft tissue surrounding the ACL was removed, and the native ACL was exposed. Then, the native ACL was removed from the insertion sites by sharp dissection. Bone tunnel at $2.5 \mathrm{~mm}$ in diameter was created with a drill in femur and tibia insertion sites of native ACL. Contralateral semitendinosus tendon was harvested and wrapped by PCL/nHAp/Col nanofiber membrane. Semitendinosus tendon wrapped with PCL membrane was used as the control group. The prepared graft was passed through the bone tunnel and joint cavity. Both ends of the graft were attached to the adjacent periosteum and soft tissue with two 4-0 Ethibond sutures (Johnson \& Johnson, New Brunswick, NJ, USA), and the wound was then closed. The rabbits were kept in the large cages without influencing their moving after surgical procedure to prevent joint stiffness. After 4 and 8 weeks postsurgery, the rabbits were sacrificed to harvest tissue samples for histological and biomechanical analysis. The interface healing between engineered tendon and host tissue was also examined.

\section{Mechanical evaluation}

Mechanical testing was performed using an electronic universal materials testing system machine (AGS-X, Shimadzu, Co., Kyoto, Japan). The specimens were harvested from 
each knee after sacrifice and prepared for mechanical testing immediately without being frozen. All soft tissues, except for the ACL graft, were carefully removed from the femurgraft-tibia complex. Before the tensile test, the samples were preloaded with a static preload of $1 \mathrm{~N}$ for 5 minutes. After preconditioning, ultimate failure load test was carried out on an elongation rate of $2 \mathrm{~mm} / \mathrm{min}$. The load-deformation curve was recorded, from which the ultimate failure load $(\mathrm{N})$ was measured. Stiffness (N/mm) was calculated from the slope of the linear region of the load-deformation curve at the maximal load-to-failure point. For each sample, the test was completed when the graft was pulled out of the bone tunnel. The results are calculated from three independent samples.

\section{Histological examination}

Immediately after sacrifice, all samples were fixed in $10 \%$ neutral buffered formalin for 48 hours, and then dehydrated through an alcohol gradient $(30 \%-100 \%)$, cleaned, and embedded in paraffin wax. The samples were sectioned at a thickness of $5 \mu \mathrm{m}$ perpendicular to the longitudinal axis of the graft with freezing microtome (SM2500, Leica, Nussloch, Germany). These sections were stained with either hematoxylin and eosin stain or Masson's trichrome stain for histological evaluation. The graft-bone interface was visualized by inverted light microscopy (IX71SBF2; Olympus Optical Co., Tokyo, Japan) and digital images were then analyzed for the bone-tissue interface with a DP Manager (Olympus Optical Co., Tokyo, Japan).

\section{Statistical analysis}

Data were expressed as the mean \pm standard deviation from three to four samples. Student's $t$-test was performed to assess statistically significant difference in the results of different experimental groups. Statistical analysis was carried out with SPSS Statistics 18.0 statistical software package (SPSS Inc., Chicago, IL, USA), and $P<0.05$ was considered to be statistically significant.

\section{Results \\ Preparation and characteristics of nanofiber membrane}

In this work, PCL/nHAp/Col nanofiber membrane was fabricated by electrospinning method. ${ }^{14} \mathrm{We}$ found that, by using the composition mass ratio of $6 / 4 / 3, \mathrm{PCL} / \mathrm{nHAp} / \mathrm{Col}$ nanofiber could be practically obtained. Figure 2 shows representative image of as-prepared nanofibers observed under SEM. It can be seen clearly that PCL/nHAp/Col nanofiber is oriented randomly with the diameter of $500 \pm 120 \mathrm{~nm}$ (Figure 2A), which is comparable to that of PCL nanofiber $(610 \pm 140 \mathrm{~nm})$. In comparison with PCL nanofiber without doping (Figure 2B), PCL/nHAp/Col nanofiber displayed coarser surface morphology. Probably, the doping of Col and nHAp into PCL might cause this outcome. This point is also supported by attenuated total reflection Fourier-transform infrared analysis. As shown in Figure 3, the signal at $1,090 \mathrm{~cm}^{-1}$ indicated stretching band of phosphate of nHAp in PCL/nHAp/Col. However, this signal was not detected in the spectra of PCL. Another two signals at 1,540 and $1,640 \mathrm{~cm}^{-1}$, found in spectra of PCL/nHAp/Col, contributed to amino group in Col. Moreover, in both PCL/nHAp/Col and PCL nanofiber, the signal at $1,720 \mathrm{~cm}^{-1}$, corresponding to the stretching of carbonyl group, could be observed. Energy spectrum analysis again revealed the presence of nitrogen atom in PCL/nHAp/Col but not in PCL nanofiber (Figure S2). These data thus reflect successful generation of PCL/nHAp/Col nanofiber. PCL/nHAp/Col membrane had
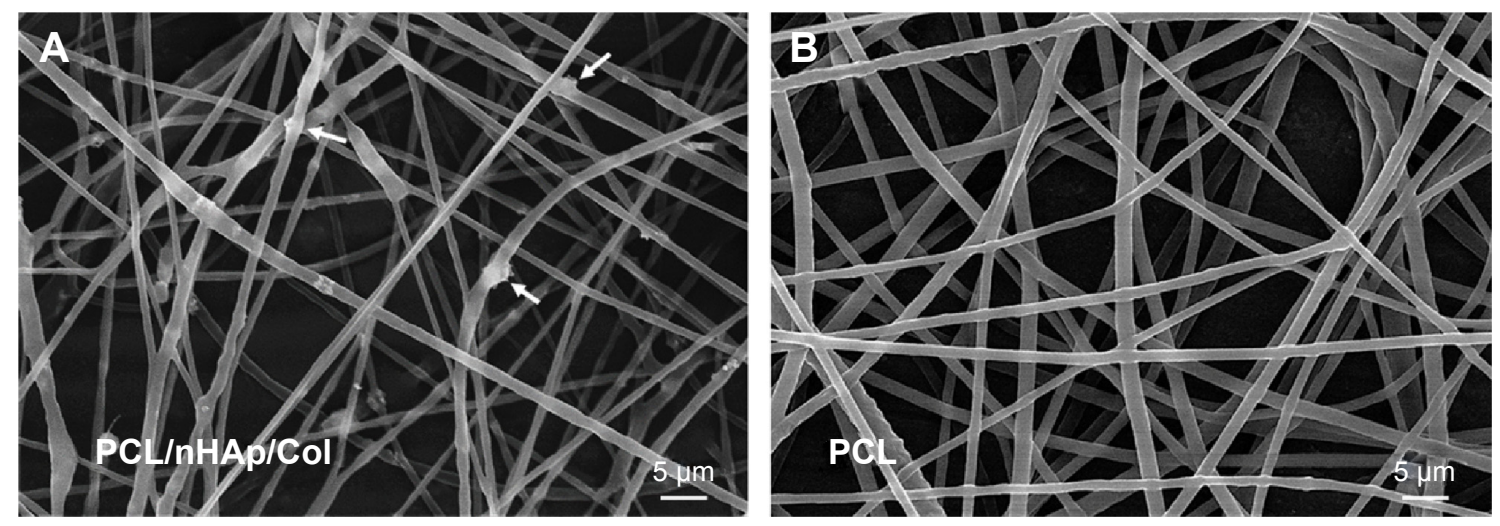

Figure 2 SEM imaging of nanofiber membranes.

Notes: (A) PCL/nHAp/Col nanofiber membrane and (B) PCL nanofiber membrane. The white arrows in $\mathbf{A}$ indicate nanohydroxyapatite.

Abbreviations: Col, collagen; $\mathrm{nHAp}$, nanohydroxyapatite; $\mathrm{PCL}$, polycaprolactone; SEM, scanning electron microscopy. 


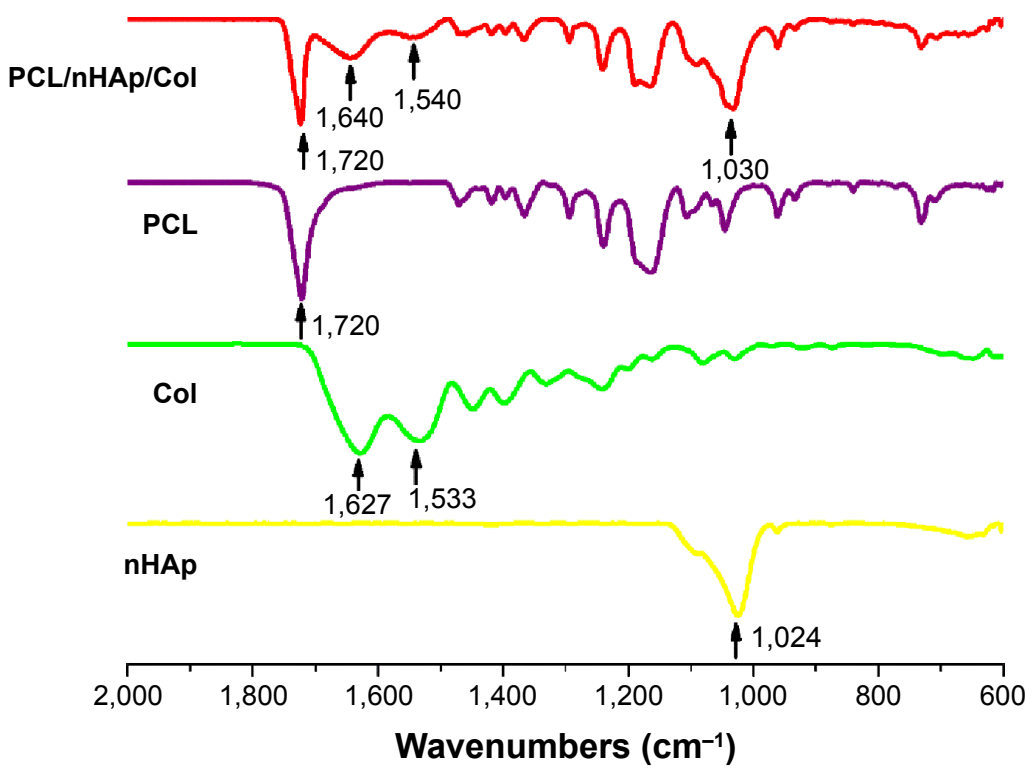

Figure 3 ATR-FTIR spectrum of PCL/nHAp/Col nanofiber, PCL nanofiber, collagen, and nHAp.

Abbreviations: ATR-FTIR, attenuated total reflection Fourier-transform infrared meter; Col, collagen; nHAp, nanohydroxyapatite; PCL, polycaprolactone.

similar pore structure with PCL membrane in terms of pore size and porosity (pore size: PCL/nHAp/Col vs PCL: 7-40 vs 5-35 $\mu \mathrm{m}$ and porosity: PCL/nHAp/Col vs PCL: $83.2 \% \pm 3 \%$ vs $75.7 \% \pm 3 \%$ ), implying that integration of $\mathrm{nHAp}$ and $\mathrm{Col}$ into PCL had a minor effect on pore structure profiles of membrane. Since high porosity and pore size are favorable for cells to obtain nutrients and excrete their metabolites, these features show high feasibility of $\mathrm{PCL} / \mathrm{nHAp} / \mathrm{Col}$ membrane for tissue engineering application.

\section{Cytocompatibility of PCL/nHAp/Col nanofiber membrane}

MC3T3 osteoblast cells were cultured in PCL/nHAp/ Col nanofiber membrane and their adhesion, growth, and mineralization behavior were investigated. SEM imaging showed that the cells displayed good adhesion (fibroblastlike) morphology in PCL/nHAp/Col nanofiber membrane and PCL membrane (Figure 4). Although the cells in the two membranes and in tissue culture plate (data not shown) showed fibroblast-like morphology within 1-7 days culture, cellular proliferation and mineralization in these membranes were rather different. Cell Counting Kit-8 assay showed that MC3T3 cells showed 1.2-fold higher growth rate in PCL/ nHAp/Col membrane than in PCL membrane or cell culture plate 7 days after cell culture (Figure $5 \mathrm{~A}$ ). This result reflects that PCL/nHAp/Col membrane is able to promote cell growth without cytotoxicity. This indicates that the presence of $\mathrm{Col}$ and nHAp into PCL membrane may enhance activity of the cells. ARS staining further showed that, at 5- and 10-day culture, a higher level of calcium deposition was found in $\mathrm{PCL} / \mathrm{nHAp} / \mathrm{Col}$ membrane compared with PCL membrane or cell culture plate, manifesting that PCL/nHAp/Col membrane is able to augment osteoblast cells mineralization (Figure 5B).

\section{$\mathrm{PCL} / \mathrm{nHAp} / \mathrm{Col}$ nanofiber membrane for tendon-bone healing}

To confirm feasibility of PCL/nHAp/Col nanofiber membrane for the healing of tendon-bone, an autologous tendon was wrapped with the nanofiber membrane and then implanted into the tunnel of the femur and tibia bone by surgery in a rabbit model. As a control, an autologous tendon without membrane treatment was also used in the same surgery. The interface between autologous tendon graft and host bone was observed by hematoxylin and eosin and Masson's staining 4 and 8 weeks after the surgery. It was found that inflammatory cells were observed that infiltrated into the graft-host bone interface 4 weeks after surgery, but the cells almost disappeared after 8 weeks (Figure 6). Besides, the thickness of fibrous scar tissue between host bone and tendon became gradually narrow from week 4 to 8 . Notably, the scar tissue thickness was clearly smaller in the PCL/ nHAp/Col group compared with the control group 8 weeks after the surgery (Figure 6), indicative of good integration between the graft and host bone. Moreover, protruding new bone tissue could be seen at the interface in $\mathrm{PCL} / \mathrm{nHAp} / \mathrm{Col}$ group by Col (Masson's) staining (Figure 7), reflecting bone formation in the interface. 

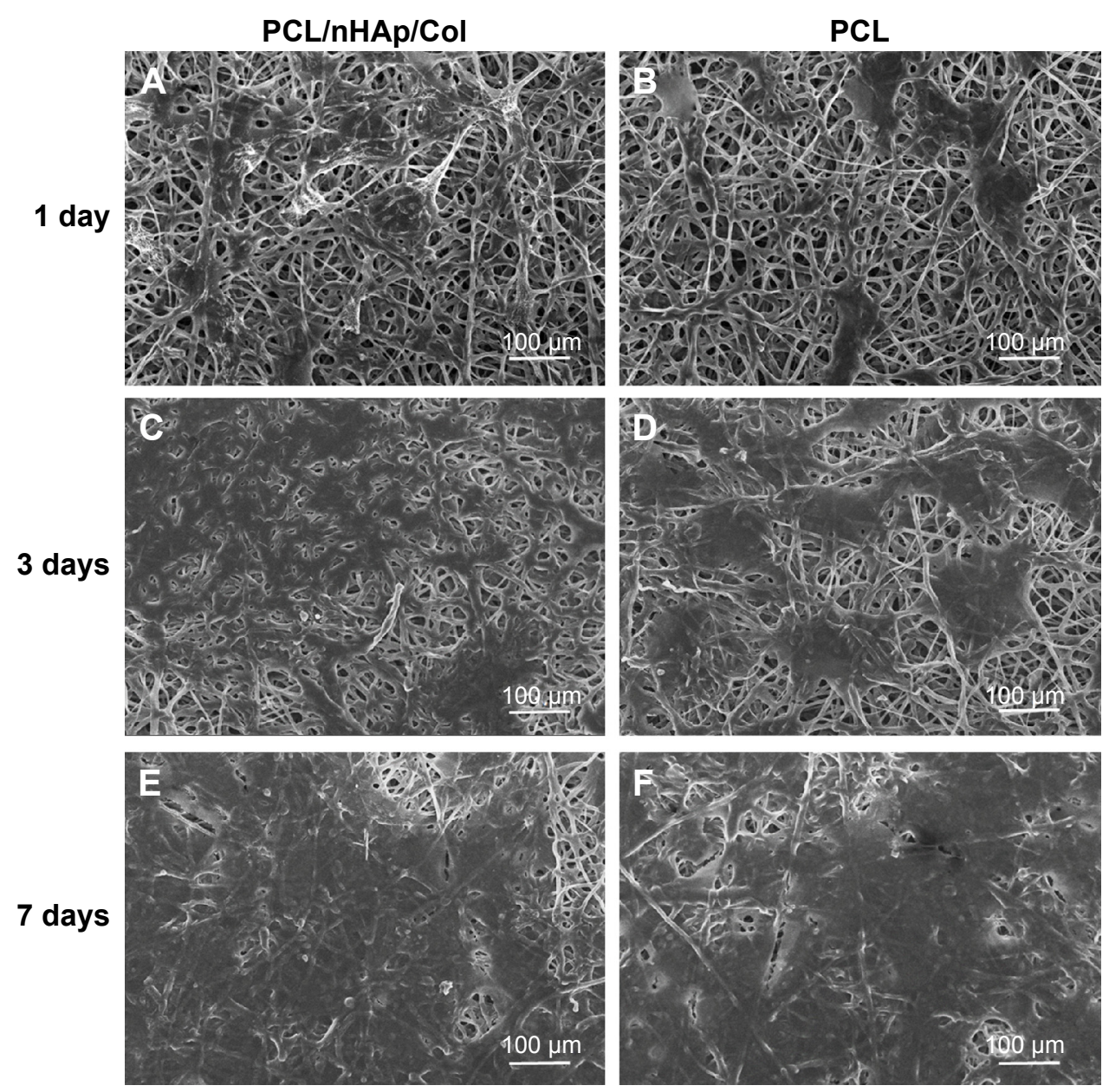

Figure 4 SEM imaging of MC3T3 cells cultured in PCL/nHAp/Col membrane or PCL membrane at I, 3, and 7 days.

Notes: (A, C, and E) Typical images of MC3T3 cells in PCL/nHAp/Col membrane. (B, D, and F) Typical images of MC3T3 cells in PCL membrane.

Abbreviations: Col, collagen; nHAp, nanohydroxyapatite; PCL, polycaprolactone; SEM, scanning electron microscopy.
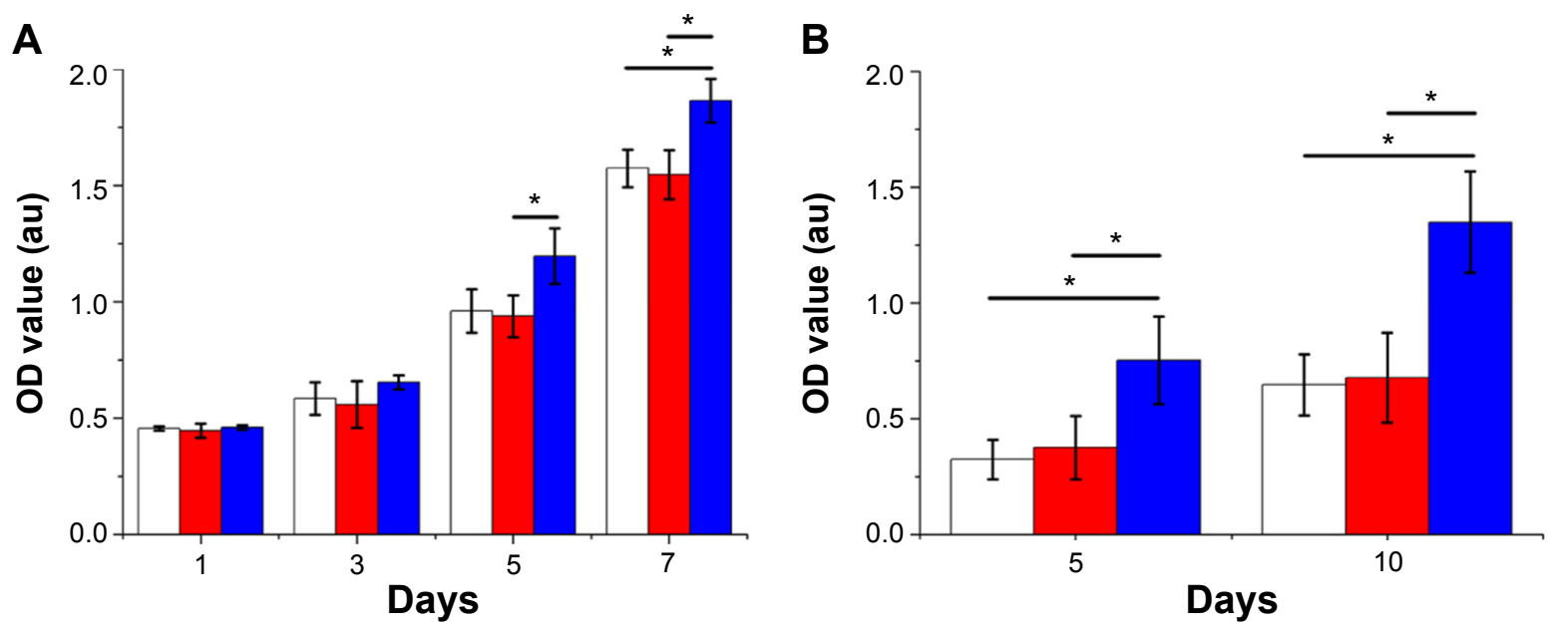

Tissue culture plastic

PCL

$\mathrm{PCL} / \mathrm{nHAp} / \mathrm{Col}$

Figure 5 Bioassay of proliferation and mineralization level of MC3T3 cells cultured in PCL/nHAp/Col membrane, PCL membrane, and culture plate as a control.

Notes: (A) CCK-8 assay showing MC3T3 proliferation at different time intervals. (B) Alizarin Red S staining showing mineral deposition level in MC3T3 at different time intervals. $* P<0.01, \mathrm{n}=5$.

Abbreviations: CCK-8, Cell Counting Kit-8; Col, collagen; nHAp, nanohydroxyapatite; PCL, polycaprolactone; OD, optical density. 

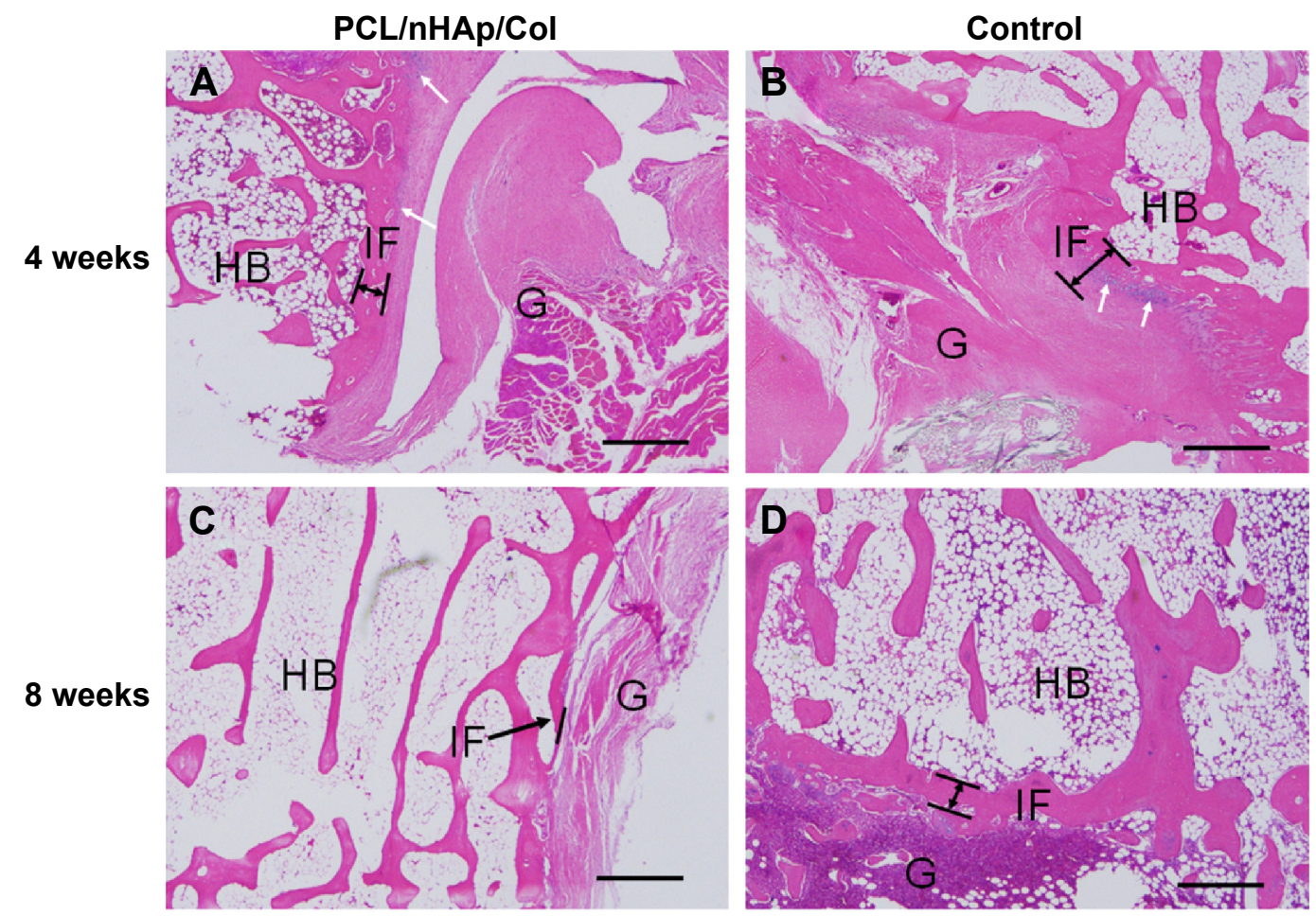

Figure 6 Histological visualization of interface between autologous tendon and host bone 4 or 8 weeks after ACL surgery in a rabbit model.

Notes: (A and C) PCL/nHAp/Col-treated tendon group. (B and D) Non-treated autologous tendon group as a control. (H\&E staining, $\times 200$ magnification). Some inflammatory cells were observed at 4 weeks (white arrows). In PCL/nHAp/Col group, new bone growth into the interface was observed 8 weeks after surgery (black arrows). Scale bar $=200 \mu \mathrm{m}$.

Abbreviations: $\mathrm{ACL}$, anterior cruciate ligament; $\mathrm{Col}$, collagen; $\mathrm{H \& E}$, hematoxylin and eosin; $\mathrm{HB}$, host bone; $\mathrm{G}$, autologous tendon graft; $\mathrm{IF}$, interface; $\mathrm{nHAp}$, nanohydroxyapatite; $\mathrm{PCL}$, polycaprolactone.

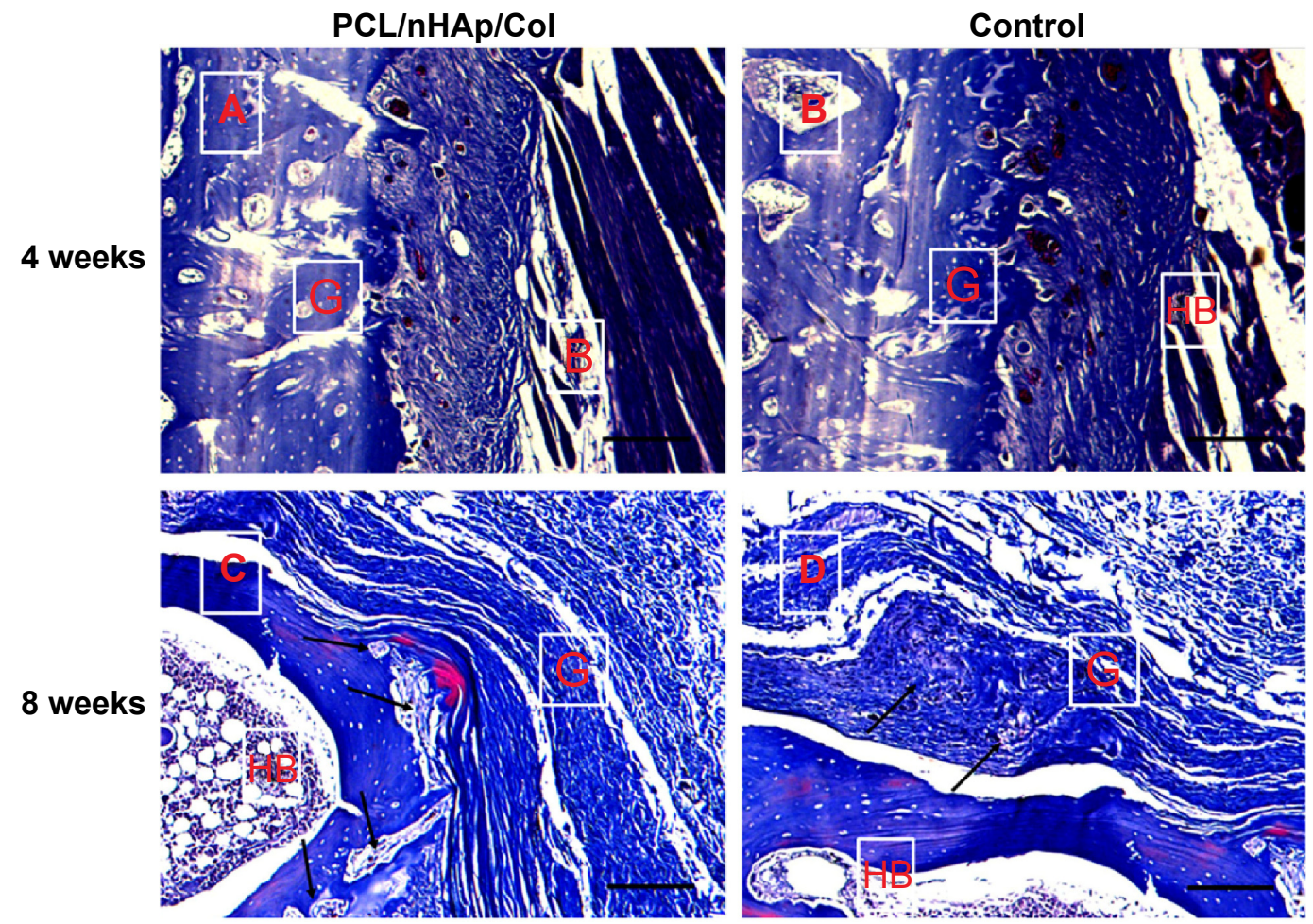

Figure 7 Masson's stain of interface between autologous tendon and host bone 4 or 8 weeks after ACL surgery in a rabbit model.

Notes: (A and C) PCL/nHAp/Col-wrapped tendon group; (B and D) PCL-wrapped autologous tendon group as a control ( $\times 40$ magnification). The formation and infiltration of the collagen fibers and bone formation at the interface of the graft and the host bone was observed 8 weeks after surgery (arrows). Scale bars $=200 \mu \mathrm{m}$. Abbreviations: $\mathrm{ACL}$, anterior cruciate ligament; Col, collagen; $\mathrm{HB}$, host bone; G, autologous tendon graft; nHAp, nanohydroxyapatite; PCL, polycaprolactone. 


\section{A}

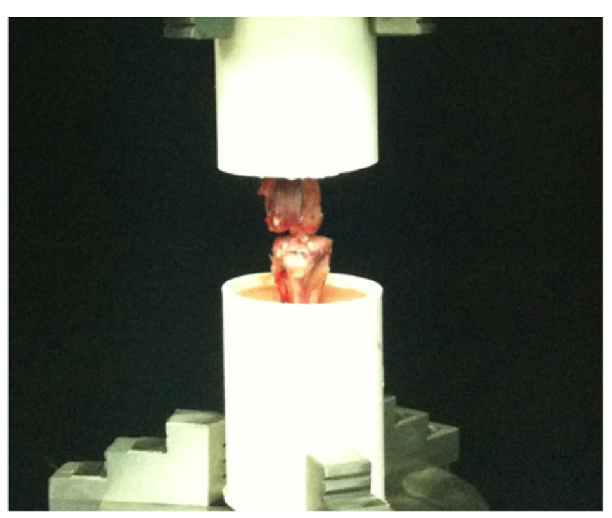

C

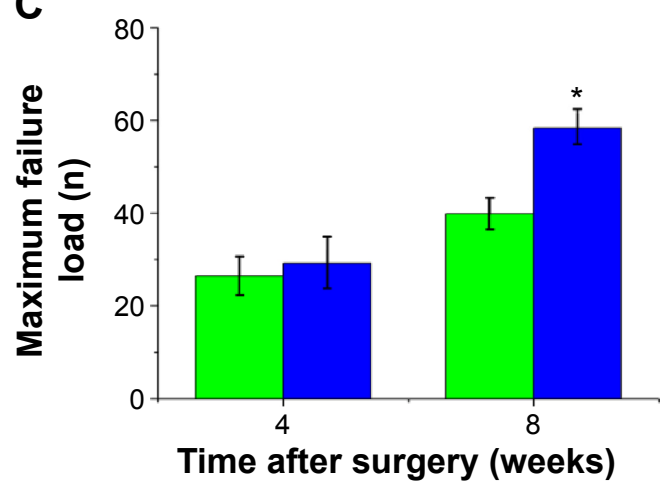

B

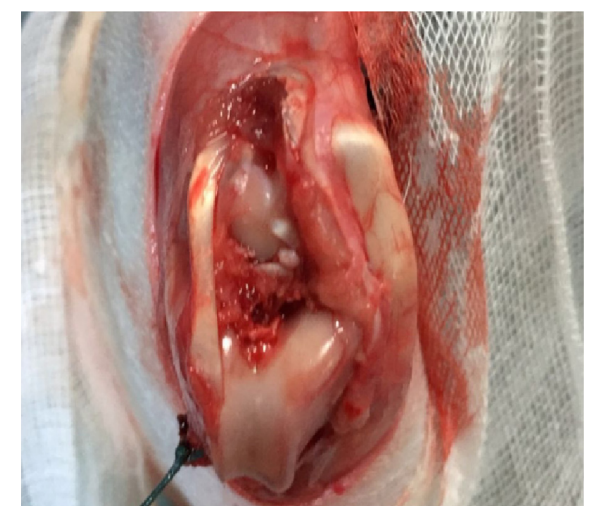

D

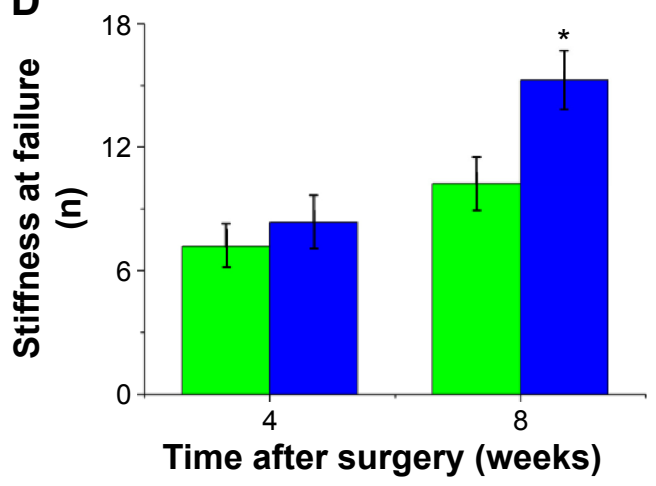

$\square$ Control $\square \mathrm{PCL} / \mathrm{nHAp} / \mathrm{Col}$

Figure 8 Biomechanical test of autologous tendon implanted 4 or 8 weeks after ACL surgery in a rabbit model.

Notes: (A) Digital picture of biomechanical test experiment of implanted tendon. (B) Autologous tendon implanted into femur and tibia bone. (C and D) the values of maximal failure load and stiffness at failure of the tendons after $\mathrm{ACL}$ surgery in $\mathrm{PCL} / \mathrm{nHAp} / \mathrm{Col}$ group and control groups. *P<0.00I; $\mathrm{n}=3$.

Abbreviations: $\mathrm{ACL}$, anterior cruciate ligament; Col, collagen; $\mathrm{nHAp}$, nanohydroxyapatite; $\mathrm{PCL}$, polycaprolactone.

All autologous tendons wrapped with $\mathrm{PCL} / \mathrm{nHAp} / \mathrm{Col}$ membrane and PCL membranes were evaluated in terms of their mechanical strength after implanted in the rabbits (Figure 8A and B). These tendons failed to be pulled out of the tunnel 4 or 8 weeks after surgery and no rupture occurred. At 4 weeks after surgery, there was no significant difference in maximum failure load between PCL/nHAp/Col group and PCL control group. However, at week 8, the failure load was significantly higher in PCL/nHAp/Col group than in PCL control group (PCL/nHAp/Col vs PCL group: $58.4 \pm 4.1$ vs $39.9 \pm 3.4 \mathrm{~N}$ ) (Figure 8C). At this time point, the average stiffness was $\sim 1.5$ times higher in PCL/nHAp/Col group than PCL group (PCL/nHAp/Col vs PCL: $15.2 \pm 1.4$ vs $10.2 \pm 1.3 \mathrm{~N} / \mathrm{mm}$ ) (Figure 8D). In addition, the membrane had not been seen in pulled-out autograft and the bone tunnel, which means in vivo degradation of the membrane. These results thus showed that $\mathrm{PCL} / \mathrm{nHAp} / \mathrm{Col}$ nanofiber membrane is highly efficient for promoting tendon-bone interface healing in vivo.

\section{Discussion}

The rupture of ACL, a main stabilizing structure of the knee, is one of the most devastating knee injuries leading to osteoarthritis. ${ }^{20}$ The hamstring autograft has been widely used as the primary graft of choice in ACL reconstruction due to low levels of postoperative pain, low comorbidity, and fewer kneeling problems. ${ }^{2}$ However, one of the major disadvantages is that hamstring autograft needs more than 3 years for sufficient integration (healing) with host bone. ${ }^{21}$ This clinical issue is ascribed to the fact that the fibroblasts instead of osteoblast proliferate more quickly in tendon-bone interface. ${ }^{21}$ This issue may be addressed by using functional membrane materials which are capable of promoting osteoregeneration in this interface.

PCL represents a biodegradable and biocompatible polymer often used for bone tissue engineering. It is a suitable candidate for fabricating fibrous scaffolds, which has been approved by the US Food and Drug Administration for various medical applications. HAp is the major mineral constituent in human bone. ${ }^{22,23}$ HAp can promote new bone growth through osteoconduction mechanism without causing any local or systemic toxicity, inflammation, or foreign body response, and is therefore suitable for bone substitution and reconstruction. ${ }^{24,25} \mathrm{nHAp}$, mimicking hydroxyapatite crystals in natural human bone tissues, is regarded to be more efficient 
for protein absorption and cell adhesion when compared with microsized hydroxyapatite materials. ${ }^{26,27}$ Incorporation of nHAp and Col into PCL fibers would provide a biomimetic scaffold with biocompatibility and good mechanical properties for osteoregeneration. Accordingly, PCL/nHAp/Col nanofiber membrane is generated in this work and used as a candidate for promoting tendon-bone healing.

As expected, PCL/nHAp/Col nanofiber membrane displays good cytocompatibility in vitro and allows for MC3T3 adhesion, proliferation, and mineralization, as a result of a large surface as well as interconnective pore structure, resembling natural bone extracellular matrix. The nHAp and Col in the membrane further serve as biological microenvironments to prompt proliferation and mineralization of host osteogenic cells or stem cells..$^{24,27}$ The Col can support the cells for good adhesion and nHAp may act as a chelating agent for mineralization of the cells. ${ }^{28}$ Therefore, in vitro studies showed that osteofibroblast cells form a cell sheet with good adhesion on the surface of PCL/nHAp/Col membrane. Besides, this membrane may induce promoted proliferation and mineralization of osteoblast cells, thereby being capable of affording the generation of new bone in the bone-tendon interface.

In vivo study further corroborates the significance of $\mathrm{PCL} / \mathrm{nHAp} / \mathrm{Col}$ nanofiber membrane for successful healing between the tendon and host bone. The histological results show that, at week 8 after surgery in a rabbit model, protruding new bone tissue form between autologous tendon and the host bone when the membrane was wrapped around the tendon. By contrast, a thick fibrous tissue was detected at the interface in the control group. This is likely ascribed to the fact that biodegradable osteomimetic nanofiber membrane can promote bone tissue infiltration into tendon-bone interface and osteogenesis. This fact also rationally interprets the reason why the mechanical property (maximum failure load and stiffness at failure) was significantly improved when using PCL/nHAp/Col membrane. The results are of clinical significance for autologous tendon healing with host bone in ACL reconstruction.

\section{Conclusion}

We have demonstrated that biomimetic PCL/nHAp/Col nanofiber membrane can provide mechanical stability, support cell proliferation, and promote osteogenesis and mineralization of MC3T3 osteoblast cells. This membrane can be applied successfully for promoting autologous tendon-bone healing in vivo. Due to good biocompatibility and biodegradability, $\mathrm{PCL} / \mathrm{nHAp} / \mathrm{Col}$ nanofiber membrane has great potential for further clinical translation in ACL reconstruction.

\section{Acknowledgments}

This work was supported by the National Basic Research Program of China (973 program) (2014CB964600 and 2012CB966300), National Natural Science Foundation of China (81271958 and 81472142), and Shanghai Municipal Natural Science Foundation (13ZR1443600).

\section{Disclosure}

The authors report no conflicts of interest in this work.

\section{References}

1. Sutton KM, Bullock JM. Anterior cruciate ligament rupture: differences between males and females. J Am Acad Orthop Surg. 2013; 21(1):41-50.

2. Murawski CD, van Eck CF, Irrgang JJ, Tashman S, Fu FH. Operative treatment of primary anterior cruciate ligament rupture in adults. $J$ Bone Joint Surg Am. 2014;96(8):685-694.

3. Hewett TE, Di Stasi SL, Myer GD. Current concepts for injury prevention in athletes after anterior cruciate ligament reconstruction. Am J Sports Med. 2013;41(1):216-224.

4. Magnussen RA, Granan LP, Dunn WR, et al. Cross-cultural comparison of patients undergoing ACL reconstruction in the United States and Norway. Knee Surg Sports Traumatol Arthrosc. 2010;18(1):98-105.

5. Ibrahim SA, Al-Kussary IM, Al-Misfer AR, Al-Mutairi HQ, Ghafar SA, El Noor TA. Clinical evaluation of arthroscopically assisted anterior cruciate ligament reconstruction: patellar tendon versus gracilis and semitendinosus autograft. Arthroscopy. 2005;21(4):412-417.

6. Keays SL, Bullock-Saxton JE, Keays AC, Newcombe PA, Bullock MI. A 6-year follow-up of the effect of graft site on strength, stability, range of motion, function, and joint degeneration after anterior cruciate ligament reconstruction: patellar tendon versus semitendinosus and Gracilis tendon graft. Am J Sports Med. 2007;35(5):729-739.

7. Font Tellado S, Balmayor ER, Van Griensven M. Strategies to engineer tendon/ligament-to-bone interface: biomaterials, cells and growth factors. Adv Drug Deliv Rev. 2015.

8. Sahoo S, Toh SL, Goh JC. A bFGF-releasing silk/PLGA-based biohybrid scaffold for ligament/tendon tissue engineering using mesenchymal progenitor cells. Biomaterials. 2010;31(11):2990-2998.

9. Shao HJ, Lee YT, Chen CS, Wang JH, Young TH. Modulation of gene expression and collagen production of anterior cruciate ligament cells through cell shape changes on polycaprolactone/chitosan blends. Biomaterials. 2010;31(17):4695-4705.

10. Shen W, Chen X, Hu Y, et al. Long-term effects of knitted silk-collagen sponge scaffold on anterior cruciate ligament reconstruction and osteoarthritis prevention. Biomaterials. 2014;35(28):8154-8163.

11. Ladd MR, Lee SJ, Stitzel JD, Atala A, Yoo JJ. Co-electrospun dual scaffolding system with potential for muscle-tendon junction tissue engineering. Biomaterials. 2011;32(6):1549-1559.

12. Fan H, Liu H, Wong EJ, Toh SL, Goh JC. In vivo study of anterior cruciate ligament regeneration using mesenchymal stem cells and silk scaffold. Biomaterials. 2008;29(23):3324-3337.

13. Shi P, Teh TK, Toh SL, Goh JC. Variation of the effect of calcium phosphate enhancement of implanted silk fibroin ligament bone integration. Biomaterials. 2013;34(24):5947-5957.

14. Wang SH, Zhao P, Lin C, Huang YL. In vitro and in vivo osteoconductivity of bone marrow stromal cells in biomimetic polycaprolactone/ calcium phosphate cement composites. J Biomater Tissue Eng. 2013;3(5):512-522.

15. Rameshbabu N, Rao KP, Kumar TSS. Accelerated microwave processing of nanocrystalline hydroxyapatite. J Mater Sci. 2005;40(23): 6319-6323.

16. Deitzel JM, Kleinmeyer J, Harris D, Tan NCB. The effect of processing variables on the morphology of electrospun nanofibers and textiles. Polymer. 2001;42(1):261-272. 
17. Jena A, Gupta K. Pore structure characterization techniques. Am Ceram Soc Bull. 2005;84(3):28-30.

18. Barhate RS, Loong CK, Ramakrishna S. Preparation and characterization of nanofibrous filtering media. J Memb Sci. 2006;283(1-2):209-218.

19. Gregory C, Gunn W, Peister A, Prockop D. An Alizarin red-based assay of mineralization by adherent cells in culture. Anal Biochem. 2004;329(1):77-84.

20. Kiapour A, Murray M. Basic science of anterior cruciate ligament injury and repair. Bone Joint Res. 2014;3(2):20-31.

21. Ekdahl M, Wang JHC, Ronga M, Fu FH. Graft healing in anterior cruciate ligament reconstruction. Knee Surg Sports Traumatol Arthrosc. 2008;16(10):935-947.

22. Webster TJ, Ergun C, Doremus RH, Siegel RW, Bizios R. Enhanced osteoclast-like cell functions on nanophase ceramics. Biomaterials. 2001;22(11):1327-1333.

23. Balasundaram G, Sato M, Webster TJ. Using hydroxyapatite nanoparticles and decreased crystallinity to promote osteoblast adhesion similar to functionalizing with RGD. Biomaterials. 2006;27(14):2798-2805.
24. Habibovic P, Kruyt MC, Juhl MV, et al. Comparative in vivo study of six hydroxyapatite-based bone graft substitutes. J Orthop Res. 2008;26(10):1363-1370.

25. O’Hare P, Meenan BJ, Burke GA, Byrne G, Dowling D, Hunt JA. Biological responses to hydroxyapatite surfaces deposited via a coincident microblasting technique. Biomaterials. 2010;31(3):515-522.

26. Sui G, Yang X, Mei F, et al. Poly-L-lactic acid/hydroxyapatite hybrid membrane for bone tissue regeneration. J Biomed Mater Res A. 2007;82(2):445-454.

27. Huang J, Lin YW, Fu XW, et al. Development of nano-sized hydroxyapatite reinforced composites for tissue engineering scaffolds. J Mater Sci Mater Med. 2007;18(11):2151-2157.

28. Venugopal J, Vadgama P, Kumar TSS, Ramakrishna S. Biocomposite nanofibres and osteoblasts for bone tissue engineering. Nanotechnology. $2007 ; 18(5)$ 


\section{Supplementary materials}

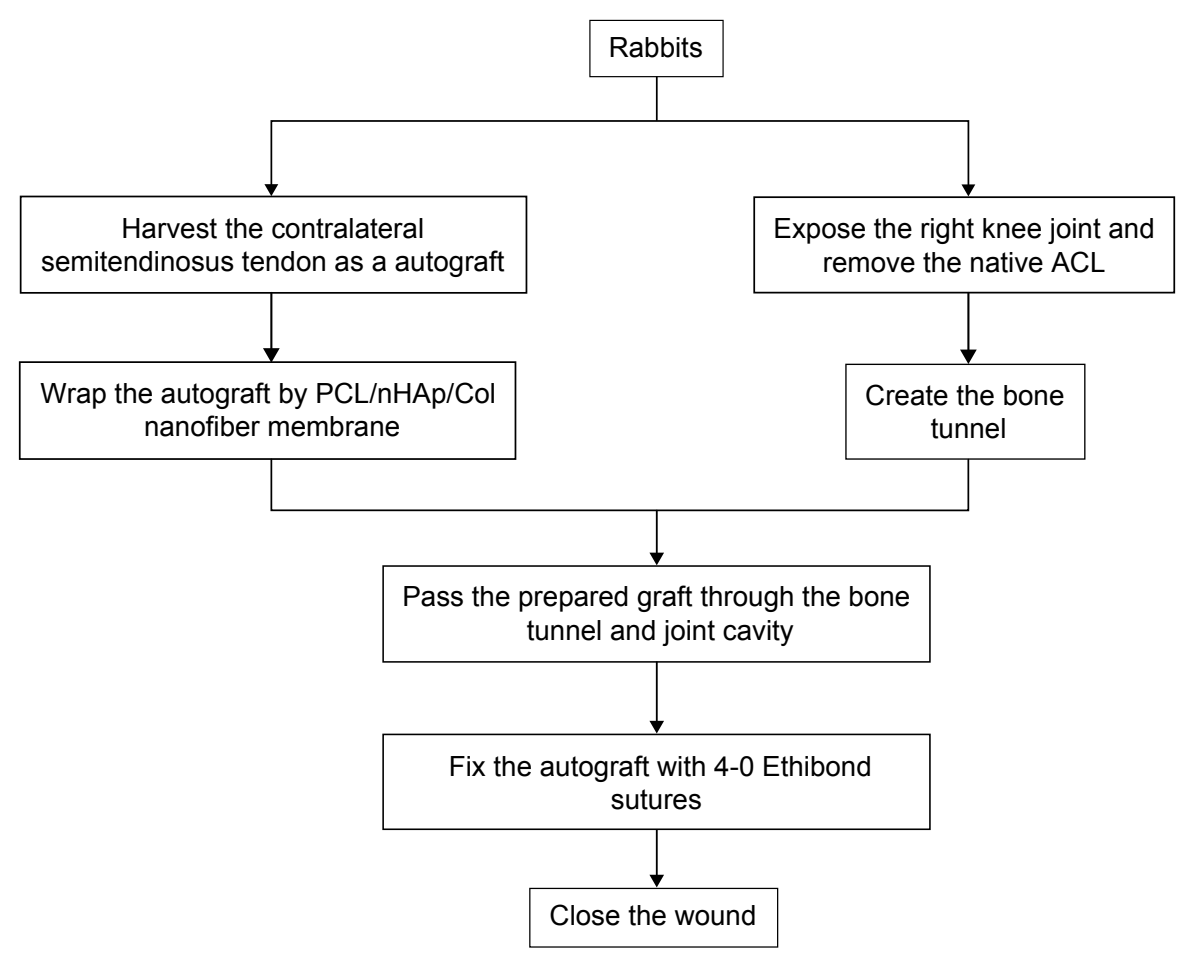

Figure SI A diagrammatic representation of the procedure on $\mathrm{ACL}$ reconstruction surgery using PCL/nHAp/Col membrane-wrapped autograft tendon. Abbreviations: $\mathrm{ACL}$, anterior cruciate ligament; Col, collagen; $\mathrm{nHAp}$, nanohydroxyapatite; $\mathrm{PCL}$, polycaprolactone.
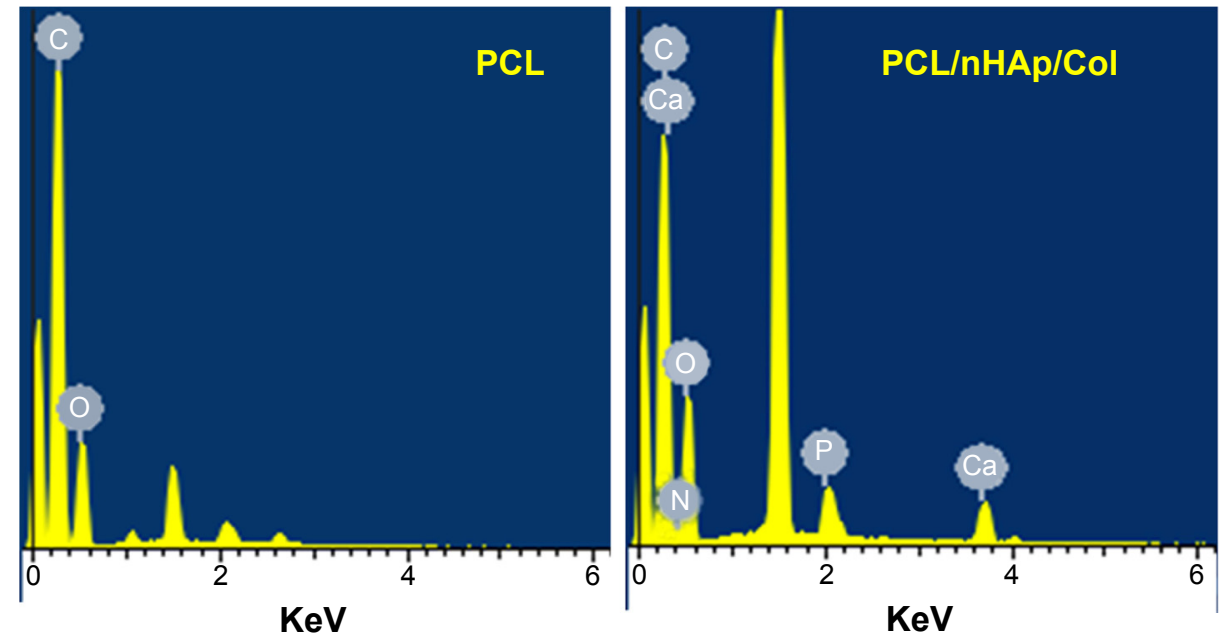

Figure S2 Energy analysis showing additional nitrogen element in $\mathrm{PCL} / \mathrm{nHAp} / \mathrm{Col}$ membrane.

Abbreviation: $\mathrm{PCL} / \mathrm{nHAp} / \mathrm{Col}$, polycaprolactone/nanohydroxyapatite/collagen.

International Journal of Nanomedicine

\section{Dovepress}

\section{Publish your work in this journal}

The International Journal of Nanomedicine is an international, peerreviewed journal focusing on the application of nanotechnology in diagnostics, therapeutics, and drug delivery systems throughou the biomedical field. This journal is indexed on PubMed Central, MedLine, CAS, SciSearch ${ }^{\circledR}$, Current Contents ${ }^{\circledR} /$ Clinical Medicine,
Journal Citation Reports/Science Edition, EMBase, Scopus and the Elsevier Bibliographic databases. The manuscript management system is completely online and includes a very quick and fair peer-review system, which is all easy to use. Visit http://www.dovepress.com/ testimonials.php to read real quotes from published authors.

Submit your manuscript here: http://www.dovepress.com/international-journal-of-nanomedicine-journal 